\title{
Circuit
}

Musiques contemporaines

\section{Premiers Kontakte}

\section{First Kontakte}

\section{Jonathan Goldman}

Volume 19, numéro 2, 2009

Stockhausen au Québec

URI : https://id.erudit.org/iderudit/037447ar

DOI : https://doi.org/10.7202/037447ar

Aller au sommaire du numéro

Éditeur(s)

Les Presses de l'Université de Montréal

ISSN

1183-1693 (imprimé)

1488-9692 (numérique)

Découvrir la revue

Citer cet article

Goldman, J. (2009). Premiers Kontakte. Circuit, 19(2), 5-9.

https://doi.org/10.7202/037447ar

\section{Résumé de l'article}

Cette présentation des articles du dossier thématique décline « Stockhausen au Québec » de trois façons différentes : les divers séjours que la personne physique de Karlheinz Stockhausen a effectués au Québec, l’influence de son oeuvre sur différentes populations de musiciens au Québec et les Québécois qui ont étudié " chez » Stockhausen. Cette introduction est complétée par une chronologie détaillée (préparée par Réjean Beaucage) des différentes visites de Stockhausen au Québec.
Ce document est protégé par la loi sur le droit d'auteur. L’utilisation des services d’Érudit (y compris la reproduction) est assujettie à sa politique d'utilisation que vous pouvez consulter en ligne.

https://apropos.erudit.org/fr/usagers/politique-dutilisation/ 


\section{INTRODUCTION AU NUMÉRO Premiers Kontakte}

Jonathan Goldman

Étudier les répercussions de la pensée de Karlheinz Stockhausen (1928-2007) sur la musique de création au Québec, c'est étudier une planète géante à partir de l'image qu'elle projette sur le miroir oblique d'un télescope. Il est parfois pratique d'observer les mouvements des planètes depuis un point de référence petit mais fixe. Disons-le tout de suite: si nous nous intéressons à la réception de l'œuvre de Karlheinz Stockhausen au Québec (et dans une moindre mesure au Canada), ce n'est pas pour nous livrer à une lecture biaisée de l'histoire - l'individu Stockhausen n'aura fait que passer brièvement chez nous sans que ces courts séjours n'influencent sa trajectoire - mais bien pour mesurer son impact de plus longue durée à partir d'une situation concrète parmi d'autres: la nôtre. En cela, la démarche de ce numéro est semblable à celle qui avait été menée à propos d'autres illustres contemporains de Stockhausen dans des numéros antérieurs de Circuit (vol. 3, n ${ }^{\circ} 1$ «Boulez au Canada»; vol. 5, $\mathrm{n}^{\circ} 2$ «Espace Xenakis»; vol. 8, $\mathrm{n}^{\circ} 2$ «Québecage»; vol. 12, n ${ }^{\circ} 1$ «Henri Pousseur: Visages»). Si c'est au courant des années 1960 que le prestige de Stockhausen a atteint un point maximal (à la suite duquel l'inévitable retour du balancier a commencé à se manifester), l'impact de son exemple s'est fait surtout sentir à partir du début des années 1970, comme les «Enquêtes» contenues ici le laisseront comprendre. Ce que nous proposons dans ce numéro qui vient souligner la disparition, en décembre 2007, de ce monstre sacré de la musique contemporaine d'expression moderniste, est donc une étude des différents reflets de la musique de Stockhausen dans l'eau québécoise. Pour ce faire, nous déclinons «Stockhausen au Québec » de trois façons différentes:

1) Stockhausen au Québec, c'est d'abord les divers séjours que la personne physique de Karlheinz Stockhausen a effectués dans la Belle Province. Une histoire en trois temps $(1958,1964,1971)$ constituant trois moments 
1. Qui, à l'occasion des 50 ans qui se sont écoulés depuis, fera l'objet d'un prochain numéro de Circuit, sous la direction de Jean Lesage.

2. Dans le prochain numéro de Circuit (vol. 19, $n^{0} 3$ ), consacré aux pionniers des studios de musique électronique au Canada, il sera beaucoup question de ce studio.
3. "Karlheinz Stockhausen, pionnier de la musique électronique, a prononcé jeudi soir à l'Université de Montréal une conférence qui est un véritable manifeste où le jeune compositeur allemand a non seulement défini la technique et l'éthique de la nouvelle forme d'expression musicale qu'il a commencé d'inventorier au studio d'essai de la radio de Cologne, mais aussi les positions de la musique dont il est le chef de file dans le contexte social et philosophique du vingtième siècle. Je n'avais pas encore entendu à Montréal, au sujet de la musique contemporaine, une thèse aussi puissamment développée, aussi scientifiquement étayée, une déclaration aussi susceptible de remettre en question toutes nos préoccupations en la matière." Jean Vallerand, "Stockhausen et la musique électronique", Le Devoir, 13 décembre 1958. distincts dans l'évolution et le développement des institutions de la musique contemporaine au Québec, que l'on pourrait symboliser par divers repères emblématiques de cette évolution : entre la première et la deuxième visite intervient la fameuse Semaine internationale de musique actuelle (1961) de Pierre Mercure' ; contemporain de sa deuxième visite (1964) est l'établissement du Studio de musique électronique de McGill, premier studio du genre au Québec ${ }^{2}$; entre la deuxième et la troisième visite est née la Société de musique contemporaine du Québec (SMCQ) (1966); c'est aussi avant sa dernière visite que voient le jour les différentes musiques in situ commandées à l'occasion de l'Expo 67 (signées entre autres par Gilles Tremblay, R. Murray Schafer, André Prévost et Otto Joachim) sans parler du Polytope de Montréal que Iannis Xenakis a érigé dans le pavillon de la France. Mais ces trois dates recoupent également trois stades relativement distincts dans la production stockhausenienne: en 1958, Stockhausen a notamment composé Gruppen, Zeitmaße, les 11 premières Klavierstücke, Kreuzspiel, le Gesang des Jünglinge, les deux études concrètes, et les premiers jets de Kontakte. C'est le Stockhausen concret et sériel. À son retour en 1964, ses œuvres récentes privilégient la mixité de sons acoustiques et électroniques: Mikrophonie I et II, Telemusik, la version intégrale de Kontakte, et, bien sûr, la bien-nommée Mixtur. Lorsque Stockhausen débarque à l'aéroport de Dorval pour entamer ce qui s'avérera être sa dernière visite à Montréal, il est l'auteur d'œuvres méditatives ou «intuitives» comme Mantra, Momente et surtout l'éblouissant Stimmung.

Ci-après une chronologie plus détaillée, préparée par Réjean Beaucage, des visites de Stockhausen au Québec qui servira à fournir un cadre temporel aux articles que vous trouverez dans ce numéro.

\section{Présences de Karlheinz Stockhausen à Montréal}

1958

11 décembre

- Enregistrement d'une entrevue pour l'émission Festivals européens, avec Maryvonne Kendergi, à la radio de Radio-Canada.

- Conférence sur «Le langage musical » à l'Université de Montréal ${ }^{3}$ (présentée par Musique de notre temps, un organisme fondé par Serge Garant, François Morel, Otto Joachim et Jeanne Landry). 
1964

28 janvier

- Concert avec Max Neuhaus et David Tudor (Zyklus, Klavierstücke VII et XI, Refrain, Kontakte). «Le Conservatoire met à notre disposition la salle Saint-Sulpice où deux concerts sont donnés. Le second doit être répété, tellement l'assistance est nombreuse.» (Maryvonne Kendergi, citée dans Louise Bail, Maryvonne Kendergi - La musique en partage, Montréal, Éditions Hurtubise HMH, 2002, p. 310).

- Conférence sur «Quatre critères de la musique électronique » au Conservatoire de musique de Montréal.

1971

2 et 3 mars

- Concert du Groupe Stockhausen à la SMCQ (Salle Claude-Champagne - Université de Montréal). Karlheinz Stockhausen, chef; Harald Bojé, électronium; Péter Eötvös, 55-chord; Aloys Kontarsky, piano.

Programme du 2 mars:

Spiral (1968), électronium solo ( $\mathrm{1}^{\mathrm{re}}$ version) ou 55-chord solo ( $2^{\mathrm{e}}$ version);

Klavierstück IX (1954-1961), piano solo;

Pole (1970), électronium et 55-chord;

Prozession (1967), électronium, piano, tam-tam, alto, deux filtres électriques et deux potentiomètres.

Programme du 3 mars:

Hymnen (1966), électronium, 55-chord, piano et tam-tam.

5 décembre

- «Musialogue» avec Maryvonne Kendergi à la Faculté de musique de l'Université de Montréal (auditions d'œuvres enregistrées, visionnement de films, discussion avec le compositeur).

6 décembre

- Concert du Collegium vocale de Cologne à la SMCQ (Théâtre Maisonneuve de la Place des Arts).

Programme: Stimmung (1968)

Dans l'un des documents de ce numéro, la première visite de Stockhausen au Québec est évoquée par un entretien radiophonique inédit. Le compositeur discute, en français, avec l'infatigable Maryvonne Kendergi, celle qui a le plus œuvré pour la diffusion et le rayonnement de la musique d'avant- 
garde au Québec. Écoutez la vigueur, l'enthousiasme avec lesquels elle présente ces sons inouïs (songez à Kontakte) à un public non averti mais curieux et vous serez étonnés par ce qu'était autrefois la radio publique canadienne. Néanmoins, 50 ans plus tard, on ne peut pas non plus rester insensible devant les troublantes métaphores de la domination (politique) explicitement véhiculées par le discours de Stockhausen. Le musicien de l'avenir, dit-il, doit apprendre la science de l'acoustique afin de «dominer» le matériau, et «il ne pourra se contenter de prendre les sonorités qu'on lui donne toutes faites, mais au contraire il refusera une dictature du matériau». Le retour du balancier anti-Stockhausen qu'on a évoqué plus haut a sûrement eu pour cause le sentiment que ce grand autocrate voulait, certes, refuser la dictature du matériau, mais en revanche imposer la sienne. Un sentiment qui, dans l'ambiance du Québec des années 1970 en quête de liberté, ne pouvait manquer de détonner.

2) Un deuxième volet de "Stockhausen au Québec » sera exploré ici : non pas la présence physique de l'homme sur le sol québécois, mais bien l'influence de son œuvre sur différentes populations de musiciens au Québec: compositeurs de musique électroacoustique, musiques improvisées/actuelles, musique savante de concert, voire de jazz ou de rock. Robert Normandeau, grâce à un questionnaire envoyé à des acteurs de la scène électroacoustique au Québec, interroge ceux-ci sur l'influence qu'a pu avoir sur eux la musique de Stockhausen, tandis que Michel F. Côté livre un témoignage tout personnel de sa rencontre avec Stockhausen, et la réaction de certains de ses confrères que l'on appelle (à tort, mais enfin) actualistes, comme le D.J. turntablist Martin Tétreault, ou le saxophoniste free Jean Derome. Une autre trace de l'influence de Stockhausen - au Canada cette fois-ci - se trouve également dans la rubrique «Document». Nous avons reproduit une conférence inédite sur Kontakte donnée à Toronto en 1968 par John Rea, alors étudiant en composition (et futur membre fondateur du comité de rédaction de Circuit); cette conférence constitue (sauf erreur, et je vous prie de nous écrire pour nous corriger, le cas échéant) le premier document analytique sur la musique de Stockhausen élaboré au Canada. En plus des intéressantes remarques analytiques qu'elle nous donne à lire, cette transcription constitue un document significatif pour quiconque voudrait mesurer l'influence du compositeur allemand sur les musiciens du Canada.

3) Un troisième et dernier volet enrichit ce numéro. «Stockhausen au Québec », c'est également les Québécois «chez» Stockhausen. Songeons déjà aux séjours, au début des années 1970, de jeunes compositeurs tels que

Walter Boudreau et Claude Vivier aux cours internationaux de Darmstadt, 
qui étaient alors essentiellement le domaine privilégié de Stockhausen et de ses condisciples. Pour certains, et en particulier pour Vivier, cette rencontre a été décisive. Nous avons demandé à Bob Gilmore, cet éminent «vivierologue » britannique, de faire état de la rencontre entre Vivier et Stockhausen. Cet article prolonge donc les réflexions sur Vivier que nous avons entreprises dans le vol. $18, n^{\circ} 3$, et nous sert à apercevoir que l'influence Vivier-Stockhausen n'aura pas toujours été à sens unique: comme le démontre Gilmore, autant le maître a influencé son disciple de façon très directe (dans les sonorités de O Kosmos!, les techniques de composition de Siddhartha, ou les textes tirés du livre occulte du XIX ${ }^{e}$ siècle Les Clavicules de Salomon choisis pour Chants), autant le maître a pu lui aussi subir l'influence de son jeune disciple (l'exemple des cloches indiennes par lesquelles se termine Inori n'est pas sans rappeler la fin de Chants). De plus, à une époque beaucoup plus récente, la flûtiste Marie-Hélène Breault a rendu visite à Stockhausen, pour travailler avec lui son œuvre Kathinkas Gesang als Lucifers Requiem qui mélange l'instrumental et l'électronique. Elle consacre le "Cahier d'analyse» de ce numéro à une étude de cette œuvre, abordée depuis son point de vue d'interprète.

Enfin, l'élément qui introduit ce dossier ne concerne pas directement le Québec. Il s'agit d'une traduction inédite en français, par les soins du musicologue Martin Kaltenecker, d'un chapitre du remarquable livre de Christoph von Blumröder Die Grundlegung der Musik Karlheinz Stockhausens (1993)4. Blumröder offre une interprétation de l'œuvre de Stockhausen à la lumière de ses croyances religieuses (catholiques) et de ses aspirations littéraires (il examine notamment un échange de lettres entre le jeune Stockhausen et son idole Hermann Hesse). Du reste, les idéaux catholiques dont le jeune Stockhausen est imprégné (même s'il les abandonne par la suite) ne sont pas sans rappeler la Weltanschauung d'un Vivier, qui baigne lui aussi dans un catholicisme mystique et tout personnel. Mais au-delà des découvertes remarquables que Blumröder décèle, il rappelle aux musicologues parmi nous une chose capitale: l'œuvre de Stockhausen est désormais clos. Il est temps que l'on en interprète le sens, que l'on produise un music criticism (au sens de Joseph Kerman5) appliqué à la musique contemporaine.

Outre le dossier Stockhausen, dans la rubrique «Actualités», Pierre Boulez et Jean-Jacques Nattiez rendent hommage au grand éditeur français Christian Bourgois, disparu quinze jours après Stockhausen, dont les activités en faveur du soutien de la musique contemporaine furent innombrables.

Bonne lecture!

Victoria, mars 2009
4. Stuttgart, Franz Steiner, 1993.

5. Une notion de music criticism que Kerman a notamment élaborée dans Contemplating Music: Challenges to Musicology, Cambridge, Harvard University Press, 1985. 